Chapter 6

\title{
An Integrated Power Flow Solution of Flexible AC Transmission Systems Containing Wind Energy Conversion Systems
}

\author{
E. Barrios-Martinez, L.M. Castro, \\ C.R. Fuerte-Esquivel and C. Angeles-Camacho \\ Additional information is available at the end of the chapter \\ http://dx.doi.org/10.5772/52835
}

\section{Introduction}

Even though the use of wind generators for converting wind energy into electricity is beneficial from the environmental standpoint, their consideration in the active power dispatch makes the already complex task of achieving power system controllability even more demanding. Consequently, the quantification of the effects that large-scale integration of wind generation will cause on the network is a very important matter that requires special attention when planning and operating an electrical power system. Arguably, power flow analysis is the most popular computational calculation performed in a power system's planning and operation, and this study has been selected to quantify the electrical response of wind generators when they are integrated in Flexible AC Transmission Systems.

Mathematical models of several types of wind generators have been developed in which their active and reactive power outputs are obtained based on the steady-state equivalent representation of the induction machine. The power injection method is then used to include these models into the power flow formulation, which is solved by using a sequential approach to obtain an operating point of the power system. In this approach, only the network's state variables are calculated through a conventional power flow algorithm, while a subproblem is formulated for updating the state variables of wind generators as well as their power injections at the end of each power flow's iteration.

Instead of using the power injection concept, another way of representing a wind generator is by means of an equivalent variable impedance expressed in terms of the slip of the generator and its rotor and stator winding parameters. This impedance is included in the system's 
admittance matrix, and the network nodal voltages are computed through the power flow analysis. Based on these voltages, the air-gap power of the wind generator is calculated and used to iteratively compute the value of the slip of the induction generator that produces the match between the air-gap power and the mechanical power extracted from the wind.

In general terms, all the methods discussed above share the characteristic of using a sequential approach to calculate the state variables of the wind generators, and none of them considers the integration of FACTS controllers in the network where the WECSs are embedded.

A fundamentally different approach for the modelling of WECS, within the context of the power flow problem, is a method that simultaneously combines the state variables associated with the wind generators, the FACTS controllers and the transmission network in a single frame-of-reference for a unified iterative solution through a Newton-Raphson (NR) technique. From the convergence standpoint, the unified method is superior to the sequential one because the interaction between the network, FACTS controllers and wind generators is better represented during the iterative solution. Furthermore, it arrives at the solution with a quadratic convergence regardless of the network size. Hence, the key contribution of this work is to provide a comprehensive and general approach for the analysis of power flows in Flexible AC Transmission Systems containing wind generators in a unified singleframe of reference.

\section{Power flow including FACTS controllers and wind generators}

The unified approach suggested in [1] is extended to compute the power flow solution of a power system containing FACTS controllers and WECS furthermore, the approach is represented by a single set of nonlinear power flow mismatch equations $f\left(X_{A C}, X_{F}, X_{W G}\right)=0$, where $X_{A C}$ is a vector of all nodal voltage magnitudes and angles, $X_{F}$ stands for the state variables of the FACTS controllers and $X_{W G}$ is a vector of all state variables associated with the wind generators. The linearised power flow mismatch equations corresponding to the wind farms are then combined with those associated with the FACTS controllers and the rest of the network, as given by (1), which are solved iteratively by the NR method:

$$
\left[\begin{array}{c}
\Delta P \\
\Delta Q \\
\Delta R_{F} \\
\Delta R_{W G}
\end{array}\right]^{j}=\left[\begin{array}{cccc}
\frac{\partial P}{\partial \theta} & V \frac{\partial P}{\partial V} & \frac{\partial P}{\partial X_{F}} & \frac{\partial P}{\partial X_{W G}} \\
\frac{\partial Q}{\partial \theta} & V \frac{\partial Q}{\partial V} & \frac{\partial Q}{\partial X_{F}} & \frac{\partial Q}{\partial X_{W G}} \\
\frac{\partial R_{F}}{\partial \theta} & V \frac{\partial R_{F}}{\partial V} & \frac{\partial R_{F}}{\partial X_{F}} & \frac{\partial R_{F}}{\partial X_{W G}} \\
\frac{\partial R_{W G}}{\partial \theta} & V \frac{\partial R_{W G}}{\partial V} & \frac{\partial R_{W G}}{\partial X_{F}} & \frac{\partial R_{W G}}{\partial X_{W G}}
\end{array}\right]^{j}\left[\begin{array}{c}
\Delta \theta \\
\frac{\Delta V}{V} \\
\Delta X_{F} \\
\Delta X_{W G}
\end{array}\right]^{j}
$$

where $\Delta R_{F}$ and $\Delta R_{W G}$ represent the mismatch equations of the FACTS controllers and wind generators, respectively. The NR method starts from an initial guess for all the state varia- 
bles and updates the solution at each iteration $i$ until a predefined tolerance is fulfilled. In this unified solution, all the state variables are adjusted simultaneously in order to compute the steady-state operating condition of the power system. Hence, this method has strong convergence characteristics.

\section{Modelling of FACTS devices}

Among all FACTS devices used to improve the steady-state performance of power systems [1], the Static Var Compensator (SVC) and Thyristor-Controlled Series Capacitor (TCSC) are the controllers considered.

\subsection{Static VAR compensator}

An integrated SVC and step-down transformer model is obtained by combining the admittances of both components $Y_{T-S V C}=Y_{T}+Y_{S V C}$ as proposed in [1]. The linearised power flow equations are given by (2) considering the firing angle $\alpha_{S V C}$ of the SVC as the state variable within the NR method

$$
\left[\begin{array}{c}
\Delta P_{k} \\
\Delta Q_{k}
\end{array}\right]=\left[\begin{array}{cc}
0 & \frac{\partial G_{T-S V C}}{\partial \alpha_{S V C}} V_{k}^{2} \\
0 & -\frac{\partial B_{T-S V C}}{\partial \alpha_{S V C}} V_{k}^{2}
\end{array}\right]\left[\begin{array}{c}
\Delta \theta_{k} \\
\Delta \alpha_{S V C}
\end{array}\right]
$$

where $G_{T-S V C}$ and $B_{T-S V C}$ are functions dependent on $\alpha_{S V C}$ :

$G_{T-S V C}=\frac{R_{T}}{R_{T}^{2}+X_{E q}^{2}}$,

$B_{T-S V C}=-\frac{X_{E q}}{R_{T}^{2}+X_{E q}^{2}}$,

$X_{E q}=X_{T}+X_{S V C}$

$X_{S V C}=\frac{X_{C} X_{T C R}}{X_{C}-X_{T C R}}$

and

$X_{T C R}=\frac{\pi X_{L}}{2\left(\pi-\alpha_{S V C}\right)+\sin \left(2 \alpha_{S V C}\right)}$. 


\subsection{Thyristor-controlled series compensator}

The TCSC firing angle power flow model is represented as an equivalent series reactance which is associated with the firing angle $\alpha_{\text {TCSC }}$. This reactance can be expressed as [1] (see Appendix):

$$
\begin{aligned}
X_{\text {TCSC }}= & -X_{C}+C_{1}\{2(\pi-\alpha)+\sin [2(\pi-\alpha)]\} \\
& -C_{2} \cos ^{2}(\pi-\alpha)\{\varpi \tan [\varpi(\pi-\alpha)]-\tan (\pi-\alpha)\}
\end{aligned}
$$

Assuming the TCSC controls the active power flowing from bus $k$ to bus $m$ to a specified value of $P_{k m}^{s p}$, the set of linearised power flow equations is

$$
\left[\begin{array}{c}
\Delta P_{k} \\
\Delta Q_{k} \\
\Delta P_{m} \\
\Delta Q_{m} \\
\Delta P_{k m}
\end{array}\right]^{j}=\left[\begin{array}{lllll}
\frac{\partial P_{k}}{\partial \theta_{k}} & \frac{\partial P_{k}}{\partial V_{k}} V_{k} & \frac{\partial P_{k}}{\partial \theta_{m}} & \frac{\partial P_{k}}{\partial V_{m}} V_{m} & \frac{\partial P_{k}}{\partial \alpha_{T C S C}} \\
\frac{\partial Q_{k}}{\partial \theta_{k}} & \frac{\partial Q_{k}}{\partial V_{k}} V_{k} & \frac{\partial Q_{k}}{\partial \theta_{m}} & \frac{\partial Q_{k}}{\partial V_{m}} V_{m} & \frac{\partial Q_{k}}{\partial \alpha_{T C S C}} \\
\frac{\partial P_{m}}{\partial \theta_{k}} & \frac{\partial P_{m}}{\partial V_{k}} V_{k} & \frac{\partial P_{m}}{\partial \theta_{m}} & \frac{\partial P_{m}}{\partial V_{m}} V_{m} & \frac{\partial P_{m}}{\partial \alpha_{T C S C}} \\
\frac{\partial Q_{m}}{\partial \theta_{k}} & \frac{\partial Q_{m}}{\partial V_{k}} V_{k} & \frac{\partial Q_{m}}{\partial \theta_{m}} & \frac{\partial Q_{m}}{\partial V_{m}} V_{m} & \frac{\partial Q_{m}}{\partial \alpha_{T C S C}} \\
\frac{\partial P_{k m}^{\alpha}}{\partial \theta_{k}} & \frac{\partial P_{k m}^{\alpha}}{\partial V_{k}} V_{k} & \frac{\partial P_{k m}^{\alpha}}{\partial \theta_{m}} & \frac{\partial P_{k m}^{\alpha}}{\partial V_{m}} V_{m} & \frac{\partial P_{k m}^{\alpha}}{\partial \alpha_{T C S C}}
\end{array}\right]\left[\begin{array}{c}
\Delta \theta_{k} \\
\Delta V_{k} \\
V_{k} \\
\Delta \theta_{m} \\
\frac{\Delta V_{m}}{V_{m}} \\
\Delta \alpha_{T C S C}
\end{array}\right]
$$

where the power flow mismatch for the TCSC module is defined as $\Delta P_{k m}=P_{k m}^{s p}-P_{k m}^{\alpha}$, $P_{k m}^{\alpha}=V_{k} V_{m} B_{k m} \sin \left(\theta_{k}-\theta_{m}\right)$ and $B_{k m}=1 / X_{T C S C}$.

\section{Modelling of wind generators}

Wind generators are categorized according to how they operate when they are connected to the grid. The Fixed-Speed Wind Generators (FSWG) are thus named so because their speed is mainly set according to the system's frequency [2]. In this category are the Stall-Regulated Fixed-Speed Wind Generators (SR-FSWG) and the Pitch-Regulated Fixed-Speed Wind Generators (PR-FSWG). A variant of the previous models is the semi-variable speed wind generator (SSWG), which uses a wound-rotor induction generator with an external resistor added to the rotor circuit in order to achieve a power regulation when wind speeds are above the rated one [3]. Also, variable-speed wind generators are being employed worldwide with the doubly-fed induction generator being the most used. However, another emergent topology that is being widely accepted is the wind generator based on a Permanent Magnet Synchronous Generator (PMSG) with a full-scale converter in which the gearbox can be omitted [4]. 
Mathematical modelling of FSWG, SSWG and PMSG-based wind generators for power flow studies is addressed below. In reference [5], the models of fixed- and semi-variable speed wind generators are suitably derived for power flow analysis and can be readily integrated in the formulation presented herein. For this reason, only a brief description of these models is given next.

\subsection{Fixed-speed wind generators}

This generator is directly connected to the network through a step-up transformer, and its final operating point depends upon the electrical frequency as well as the nodal voltage at the generator's terminals. The generated reactive and active powers are determined by Equations (5) and (6), respectively, and the stator and rotor currents of the induction generator can be expressed according to Equations (7) and (8) [5] :

$$
\begin{gathered}
Q_{g}(V, s)=-V^{2}\left[\frac{A+B s^{2}}{(C-D s)^{2}+(E+F s)^{2}}\right] \\
P_{g}(V, s)=-V^{2}\left[\frac{K+H s+L s^{2}}{(C-D s)^{2}+(E+F s)^{2}}\right] \\
I_{1}^{2}(V, s)=V^{2}\left\{\frac{\left(K+H s+L s^{2}\right)^{2}+\left(A+B s^{2}\right)^{2}}{\left[(C-D s)^{2}+(E+F s)^{2}\right]^{2}}\right\} \\
I_{2}^{2}(V, s)=V^{2}\left\{\frac{\left(M s+N s^{2}\right)^{2}+\left(T s-W s^{2}\right)^{2}}{\left[(C-D s)^{2}+(E+F s)^{2}\right]^{2}}\right\}
\end{gathered}
$$

where $s$ is the machine's slip, $V$ is the terminal voltage, and the constants from $A$ to $W$ are as given in the Appendix. Furthermore, the power converted from mechanical to electrical form $\left(P_{\text {conv }}\right)$ can be computed by using Equation (9), where $R_{2}$ represents the rotor resistance

$$
P_{\text {conv }}=-I_{2}^{2} R_{2}\left(\frac{1-s}{s}\right)
$$

\subsubsection{Stall-regulated fixed-speed wind generator}

The mechanical power $P_{m}[\mathrm{~W}]$ extracted from the wind by this generator is given by [6] 


$$
P_{m}=0.5 \cdot \rho \cdot c_{1}\left(\frac{c_{2}}{\lambda_{i}}-c_{3} \beta-c_{4} \beta^{c_{5}}-c_{6}\right) \cdot e^{\frac{-c_{7}}{\lambda_{i}}} \cdot A \cdot V_{w}^{3}
$$

where

$\lambda_{i}=\left[\left(\frac{1}{\lambda+c_{8} \beta}\right)-\left(\frac{c_{9}}{\beta^{3}+1}\right)\right]^{-1}$

and

$\lambda=\frac{R \cdot n_{g b} \cdot \omega_{S}(1-s)}{V_{w}}$

- $\rho$ is the air density $\left[\mathrm{kg} / \mathrm{m}^{3}\right]$,

- $A$ is the swept area of the blades $\left[\mathrm{m}^{2}\right]$,

- $V_{w}$ is the wind speed $[\mathrm{m} / \mathrm{s}]$,

- $R$ is the radius of the rotor [m],

- $n_{\mathrm{gb}}$ is the gearbox ratio,

- $\omega_{s}$ is the angular synchronous speed [rad/s],

- $\beta$ is the pitch angle [degrees],

- $\omega_{T}$ is the angular speed of the turbine $[\mathrm{rad} / \mathrm{s}]$,

- and the constants $c_{1}$ to $c_{9}$ are the parameters of the wind turbine's design.

Thus, assuming that the SR-FSWG is connected at bus $k$, the power mismatches equations are (11)-(13), and the set of linearised equations that has to be assembled and combined with the Jacobian matrix and the power mismatch vector of the entire network is shown in Equation (14) [5]:

$$
\begin{gathered}
\Delta P_{k}=P_{g}(V, s)-P_{L k}-P_{k}^{c a l}=0 \\
\Delta Q_{k}=Q_{g}(V, s)-Q_{L k}-Q_{k}^{c a l}=0 \\
\Delta P_{W T 1, k}=-\left\{P_{m}-P_{\text {conv }}\right\}=-\left\{P_{m}+I_{2}^{2} R_{2}\left(\frac{1-s}{s}\right)\right\}=0
\end{gathered}
$$




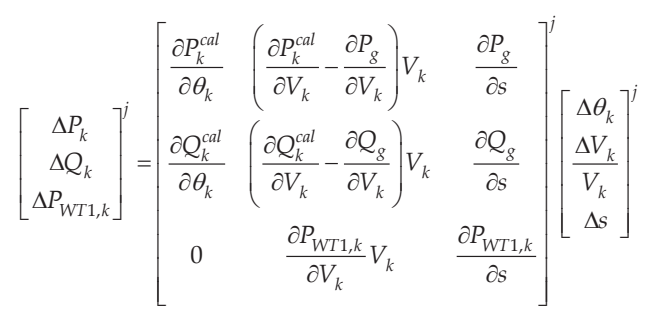

where $P_{g}(V, s)$ and $Q_{g}(V, s)$ are given by (6) and (5), respectively, $P_{L K}$ and $Q_{L K}$ represent the active and reactive powers drawn by the load at bus $k$, respectively, and $P_{k}{ }^{c a l}$ and $Q_{k}{ }^{c a l}$ are active and reactive power injections given by

$$
\begin{gathered}
P_{k}^{c a l}=V_{k}^{2} G_{k k}+V_{k} \sum_{m \in k} V_{m}\left[G_{k m} \cos \left(\theta_{k}-\theta_{m}\right)+B_{k m} \sin \left(\theta_{k}-\theta_{m}\right)\right] \\
Q_{k}^{c a l}=-V_{k}^{2} B_{k k}+V_{k} \sum_{m \in k} V_{m}\left[G_{k m} \sin \left(\theta_{k}-\theta_{m}\right)-B_{k m} \cos \left(\theta_{k}-\theta_{m}\right)\right]
\end{gathered}
$$

\subsubsection{Pitch-regulated fixed-speed wind generator}

Since this wind generator has a blade pitch angle mechanism which actuates to limit the power extracted from the wind [7], the generated active power $P_{g, p r}$ can be obtained from its power curve and is considered constant at a value $P_{g, p r}^{s p}$ through the iterative process; however, the reactive power $Q_{g, p r}=Q_{g}$ needs to be calculated [5]. Therefore, the internal power equilibrium point in the wind generator has to be computed by Equation (17):

$$
P_{m, p r}=P_{g, p r}^{s p}+P_{\text {losses }, s}+P_{l o s s e s, r}=P_{g, p r}^{s p e}+3 I_{1}^{2} R_{1}+3 I_{2}^{2} R_{2}
$$

where $P_{\text {losses,s }}$ and $P_{\text {losses, } r}$ are the three-phase stator and rotor power losses, respectively, and the core losses in the induction machine are neglected. As mentioned previously, the set of linearised power flow mismatch equations regarding the PR-FSWG is given by Equations (18)-(21) when the generator is connected at bus $k$ :

$$
\begin{gathered}
\Delta P_{k}=P_{g, p r}-P_{L k}-P_{k}^{c a l}=0 \\
\Delta Q_{k}=Q_{g}(V, s)-Q_{L k}-Q_{k}^{c a l}=0 \\
\Delta P_{W T 2, k}=-P_{g, p r}^{s p}-\left\{\left(3 I_{1}^{2} R_{1}+3 I_{2}^{2} R_{2}\right)+I_{2}^{2} R_{2}\left(\frac{1-s}{s}\right)\right\}=0
\end{gathered}
$$




$$
\left[\begin{array}{c}
\Delta P_{k} \\
\Delta Q_{k} \\
\Delta P_{W T 2, k}
\end{array}\right]^{j}=\left[\begin{array}{ccc}
\frac{\partial P_{k}^{c a l}}{\partial \theta_{k}} & \frac{\partial P_{k}^{c a l}}{\partial V_{k}} V_{k} & 0 \\
\frac{\partial Q_{k}^{c a l}}{\partial \theta_{k}} & \left(\frac{\partial Q_{k}^{c a l}}{\partial V_{k}}-\frac{\partial Q_{g}}{\partial V_{k}}\right) V_{k} & \frac{\partial Q_{g}}{\partial s} \\
0 & \frac{\partial P_{W T 2, k}}{\partial V_{k}} V_{k} & \frac{\partial P_{W T 2, k}}{\partial s}
\end{array}\right]^{j}\left[\begin{array}{c}
\Delta \theta_{k} \\
\frac{\Delta V_{k}}{V_{k}} \\
\Delta s
\end{array}\right]^{j}
$$

\subsection{Semi-variable speed wind generator}

In this type of generator, the slip of the induction machine cannot be regarded as the state variable because of the external resistance $R_{\text {ext }}$ added in the rotor circuit [5]. Hence, a total rotor circuit resistance, $R_{x}=\left(R_{2}+R_{e x t}\right) / s$, is considered as a single-state variable associated with the rotor circuit, which is adjusted to satisfy the power mismatch equations during the NR power flow calculation $[5,8]$. Hence, the reactive and active powers, the stator and rotor currents as well as the power converted from mechanical to electrical form will be dependent functions on $R_{x}$ and can be expressed as

$$
\begin{gathered}
Q_{g, s s}\left(V, R_{x}\right)=-V^{2}\left[\frac{A^{\prime} R_{x}^{2}+B}{\left(C^{\prime} R_{x}-D\right)^{2}+\left(E^{\prime} R_{x}+F\right)^{2}}\right] \\
P_{g, s s}\left(V, R_{x}\right)=-V^{2}\left[\frac{K^{\prime} R_{x}{ }^{2}+H^{\prime} R_{x}+L}{\left(C^{\prime} R_{x}-D\right)^{2}+\left(E^{\prime} R_{x}+F\right)^{2}}\right] \\
I_{1, s s}^{2}\left(V, R_{x}\right)=V^{2}\left\{\frac{\left(K^{\prime} R_{x}{ }^{2}+H^{\prime} R_{x}+L\right)^{2}+\left(A^{\prime} R_{x}^{2}+B\right)^{2}}{\left[\left(C^{\prime} R_{x}-D\right)^{2}+\left(E^{\prime} R_{x}+F\right)^{2}\right]^{2}}\right\} \\
I_{2, s s}^{2}\left(V, R_{x}\right)=V^{2}\left\{\frac{\left(M^{\prime} R_{x}+N\right)^{2}+\left(T^{\prime} R_{x}-W\right)^{2}}{\left[\left(C^{\prime} R_{x}-D\right)^{2}+\left(E^{\prime} R_{x}+F\right)^{2}\right]^{2}}\right\} \\
P_{\text {conv }}=-I_{2, s s}^{2} R_{2}\left(\frac{1-s}{s}\right) \approx-I_{2, s s}^{2} R_{2}\left(\frac{1}{s}\right)=-I_{2, s s}^{2} R_{x}
\end{gathered}
$$

where the constants $A^{\prime}, C^{\prime}, E^{\prime}, H^{\prime}, K^{\prime}, M^{\prime}$ and $T^{\prime}$ are given in the Appendix. Since the generated active power $P_{g, s s}$ is set to a fixed value $P_{g, s s}^{s p}$ obtained from the wind generator power 
curve, and assuming no core losses, the mechanical power of the induction generator $P_{m, s s}$ can be estimated as follows:

$$
P_{m, s s}=P_{g, s s}^{s p}+P_{l o s s e s, s}+P_{l o s s e s, r}=P_{g, s s}^{s p}+3 I_{1, s s}^{2} R_{1}+3 I_{2, s s}^{2} R_{2}
$$

Therefore, when the SSWG is connected at bus $k$, its set of mismatch power flow equations is

$$
\left.\begin{array}{c}
\Delta P_{k}=P_{g, s s}^{s p}-P_{L k}-P_{k}^{c a l}=0 \\
\Delta Q_{k}=Q_{g, s s}\left(V, R_{x}\right)-Q_{L k}-Q_{k}^{c a l}=0 \\
\Delta P_{W T 3, k}=-P_{g, s s}^{s p}-\left\{3 I_{1, s s}^{2} R_{1}+I_{2, s s}^{2} R_{x}\right\}=0 \\
{\left[\begin{array}{c}
\Delta P_{k} \\
\Delta Q_{k} \\
\Delta P_{W T 3, k}
\end{array}\right]^{j}=\left[\begin{array}{ccc}
\frac{\partial P_{k}^{c a l}}{\partial \theta_{k}} & \frac{\partial P_{k}^{c a l}}{\partial V_{k}} V_{k} & 0 \\
\frac{\partial Q_{k}^{c a l}}{\partial \theta_{k}} & \left.\frac{\partial Q_{k}^{c a l}}{\partial V_{k}}-\frac{\partial Q_{g, s s}}{\partial V_{k}}\right) V_{k} & \frac{\partial Q_{g, s s}}{\partial R_{x}} \\
0 & \frac{\partial P_{W T 3, k}}{\partial V_{k}} V_{k} & \frac{\partial P_{W T 3, k}}{\partial R_{x}}
\end{array}\right]^{j}} \\
\frac{\Delta V_{k}}{V_{k}} \\
\Delta R_{x}
\end{array}\right]^{j}
$$

where the internal energy balance in the induction machine $\Delta P_{W T 3}=-\left(P_{m, s s}-P_{\text {conv }}\right)$ is derived by using Equations (26)-(27).

\subsection{PMSG-based wind generator}

This type of wind generator possesses a PMSG and a full-rated converter to connect the generator to the network, resulting in complete speed and reactive power control [9]. Hence, all the generated power is supplied to the power system through a machine-side converter and gridside converter. The schematic diagram of this topology is shown in figure 1(a). Reactive power support is one of the characteristics that make this machine attractive for wind power production. In this case, the inclusion of the explicit representation of the wind generator stepup transformer is considered, which allows for direct voltage magnitude control at the highvoltage side of the transformer. The proposed model for power flow studies is shown in the figure 1(b) in which $P_{\text {g,pms }}$ represents the output power set by the wind generator power curve for a given wind speed, $V_{m s c}$ and $V_{g s c}$ are the voltage at the machine-side converter and gridside converter terminal, respectively, and $Z_{s t}$ is the step-up transformer impedance. 


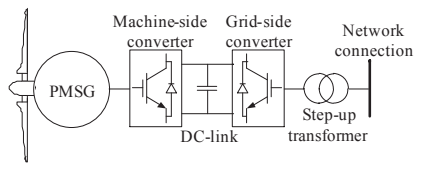

(a)

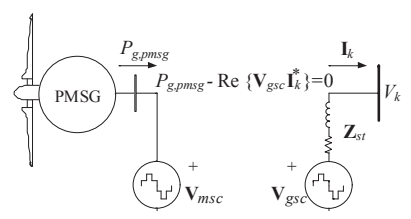

Figure 1. PMSG-based wind generator: (a) schematic diagram, (b) proposed model for power flow studies.

The power flow equations for the PMSG-based wind generator are derived assuming the following voltage at the grid-side converter terminal: $\mathrm{V}_{g s c}=V_{g s c} e^{j \delta g s c}$. Based on Fig. 1(b), the active and reactive powers flowing from the grid-side converter terminal to the $k$-th bus are

$$
\begin{gathered}
P_{g s c-k}=V_{g s c}^{2} G_{s t}+V_{g s c} V_{k}\left[G_{s t} \cos \left(\delta_{g s c}-\theta_{k}\right)+B_{s t} \sin \left(\delta_{g s c}-\theta_{k}\right)\right] \\
Q_{g s c-k}=-V_{g s c}^{2} B_{s t}+V_{g s c} V_{k}\left[G_{s t} \sin \left(\delta_{g s c}-\theta_{k}\right)-B_{s t} \cos \left(\delta_{g s c}-\theta_{k}\right)\right]
\end{gathered}
$$

For the active and reactive powers at bus $k$, the subscripts gsc and $k$ are exchanged in Equations (32) and (33). Therefore, the NR-based power flow formulation is given by

$$
\begin{aligned}
& \Delta P_{k}=-P_{k-g s c}-P_{L k}-P_{k}^{c a l}=0 \\
& \Delta Q_{k}=-Q_{k-g s c}-Q_{L k}-Q_{k}^{c a l}=0 \\
& \Delta P_{W T 4, k}=P_{g, p m s g}-P_{g s c-k}=0 \\
& {\left[\begin{array}{c}
\Delta P_{k} \\
\Delta Q_{k} \\
\Delta P_{W T 4, k}
\end{array}\right]^{j}=\left[\begin{array}{ccc}
\frac{\partial P_{k}^{c a l}}{\partial \theta_{k}} & \frac{\partial P_{k}^{c a l}}{\partial V_{g s c}} V_{g s c} & \frac{\partial P_{k-g s c}}{\partial \delta_{g s c}} \\
\frac{\partial Q_{k}^{c a l}}{\partial \theta_{k}} & \frac{\partial Q_{k}^{c a l}}{\partial V_{g s c}} V_{g s c} & \frac{\partial Q_{k-g s c}}{\partial \delta_{g s c}} \\
\frac{\partial P_{W T 4, k}}{\partial \theta_{k}} & \frac{\partial P_{W T 4, k}}{\partial V_{g s c}} V_{g s c} & \frac{\partial P_{W T 4, k}}{\partial \delta_{g s c}}
\end{array}\right]^{j}\left[\begin{array}{c}
\Delta \theta_{k} \\
\frac{\Delta V_{g s c}}{V_{g s c}} \\
\Delta \delta_{g s c}^{j}
\end{array}\right]^{-}}
\end{aligned}
$$


Note that Equation (36) represents the power constraint in the AC/DC/AC converter in which active power losses are neglected.

\section{Case studies with wind farms and FACTS devices}

This section shows how the proposed approach performs when considering a power system having FACTS devices and wind generators.

\subsection{Five-bus test system with FSWGs, SSWGs and a SVC}

The typical five-bus test system is used to provide an example with the inclusion of a wind farm consisting of ten SR-FSWGs, ten PR-FSWGs and ten SSWGs operating at a wind speed of $16 \mathrm{~m} / \mathrm{s}$ with which all wind generators are injecting their maximum power. Additionally, a SVC is placed at bus five in order maintain its terminal voltage magnitude at $1 \mathrm{pu}$. The conventional generators are set to control voltage magnitudes at $1 \mathrm{pu}$. Parameters of the wind farm are given in the Appendix.

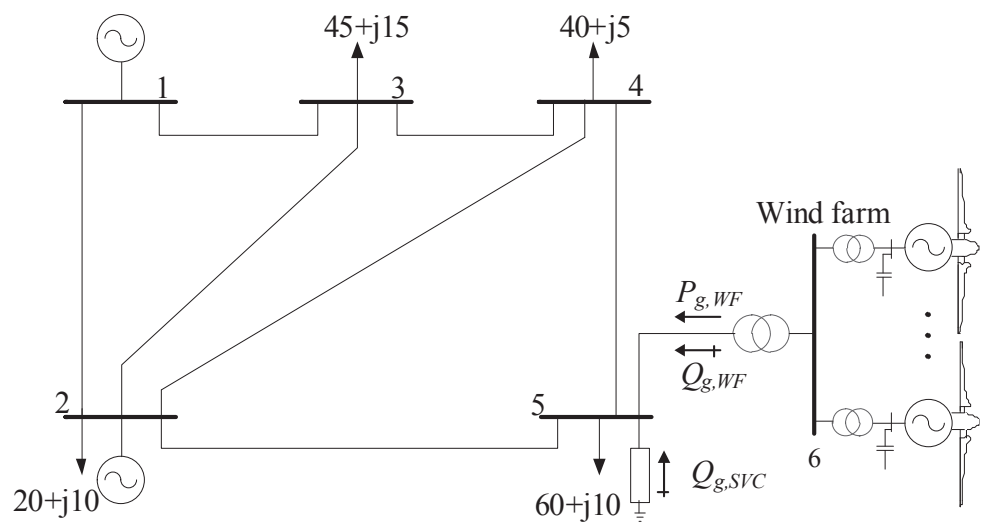

Figure 2. Modified five-bus test system used to incorporate a SVC and a wind farm composed of several wind generator models.

In order to show the effect of wind farms and a SVC in the operation of the power system, the following three scenarios are presented: (a) the base case where the wind farm and SVC are not considered, (b) the case where only the wind farm is running and (c) the case where the SVC is connected at the bus at which the wind farm is connected in order to provide voltage support. The results regarding each case are reported in Table I. 


\begin{tabular}{llll}
\hline Results & \multicolumn{3}{c}{ Scenario } \\
\cline { 2 - 4 } & (a) & (b) & (c) \\
\hline$V_{1}$ & 1.000 & 1.000 & 1.000 \\
$V_{2}$ & 1.000 & 1.000 & 1.000 \\
$V_{3}$ & 0.971 & 0.971 & 0.977 \\
$V_{4}$ & 0.971 & 0.971 & 0.979 \\
$V_{5}$ & 0.967 & 0.966 & 1.000 \\
$V_{6}$ & --- & 0.945 & 0.981 \\
$P_{g, W F}$ & --- & 25.349 & 25.341 \\
$Q_{g, W F}$ & --- & -10.802 & -9.857 \\
$Q_{g, S V C}$ & --- & --- & 36.897 \\
\hline
\end{tabular}

Table 1. Power flow simulation results with wind farm and SVC.

The simulated wind farm is a reactive power consumer since it lacks a reactive power control as seen from Table 1. When no SVC is considered, its reactive power absorption exceeds 10 MVAr, resulting in a low-voltage magnitude at node six. On the other hand, when the SVC is set in operation, not only the low-voltage side of the wind farm transformer is boosted, but also the system voltage profile.

Since all wind generators are operating at the same wind speed, clearly the state variables calculated for each group of wind generators corresponding to each model will result in the same value. Furthermore, if the system voltage profile changes, as occurs with the inclusion of the SVC, another operating point is found at each wind generator as shown in Table 2.

\begin{tabular}{ccccc}
\hline Scenario & SR-FSWG & PR-FSWG & SSWG & SVC \\
\hline s & $\mathbf{s}$ & $\mathbf{R}_{\mathbf{x}}$ & $\boldsymbol{a}_{\text {svc }}($ deg $)$ & \\
\hline (b) & -0.00506 & -0.00679 & -67.69437 & -- \\
\hline (c) & -0.00467 & -0.00613 & -75.16952 & 136.35024 \\
\hline
\end{tabular}

Table 2. Computed wind generators and SVC state variables.

\subsection{Five-bus test system with PMSG-based wind generators and a TCSC}

In this case, a PMSG-based wind farm is located at bus 4 with 30 wind generators operating at a rated wind speed, i.e. $15 \mathrm{~m} / \mathrm{s}$. Be aware that each PMSG-based wind generator provides reactive power support by controlling its terminal voltage magnitude. Also, a TCSC is placed for controlling the active power flowing through the transmission line connected between nodes 4 and 5 at $P_{4-5}=20 \mathrm{MW}$, as shown in figure 2. The next scenarios are analyzed: (a) the base case where the wind farm and TCSC are not considered, (b) the power system 
including only the PMSG-based wind farm and (c) the network having simultaneously the TCSC and the wind farm. The results are reported in Table 3.

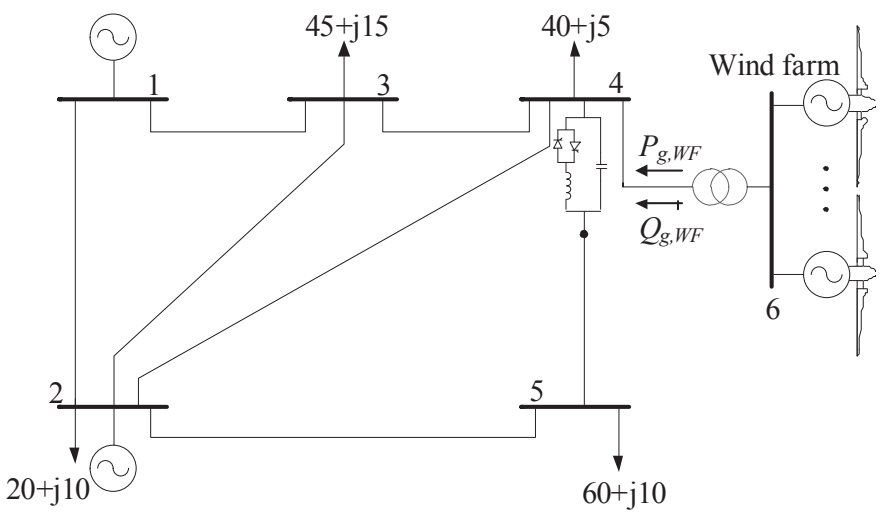

Figure 3. Modified five-bus test system used to incorporate a TCSC and a PMSG-based wind farm.

\begin{tabular}{cccc}
\hline Results & \multicolumn{3}{c}{ Scenario } \\
\hline (a) & (b) & (c) \\
\hline$V_{1}$ & 1.000 & 1.000 & 1.000 \\
\hline$V_{2}$ & 1.000 & 1.000 & 1.000 \\
\hline$V_{3}$ & 0.971 & 0.984 & 0.984 \\
\hline$V_{4}$ & 0.971 & 0.987 & 0.988 \\
\hline$V_{5}$ & 0.967 & 0.973 & 0.971 \\
\hline$V_{6}$ & --- & 1.000 & 1.000 \\
\hline$P_{g, W F}$ & --- & 60.000 & 60.000 \\
\hline$Q_{g, W F}$ & --- & 2.566 & 2.218 \\
\hline$P_{1-2}$ & 89.683 & 46.792 & 45.883 \\
\hline$P_{1-3}$ & 40.639 & 20.252 & 21.206 \\
\hline$P_{2-3}$ & 24.821 & 11.565 & 13.114 \\
\hline$P_{2-4}$ & 28.076 & 9.279 & 11.253 \\
\hline$P_{2-5}$ & 55.032 & 45.465 & 41.053 \\
\hline$P_{4-3}$ & -18.686 & 13.628 & 11.171 \\
\hline$P_{4-5}$ & 6.258 & 15.590 & 20.000 \\
\hline & & & \\
\hline
\end{tabular}

Table 3. Power flow simulation results with wind farm and TCSC. 
The system voltage profile is improved when the PMSG-based wind farm is integrated to the grid as seen from Table 3. This is mainly due to two reasons; there is a redistribution of power flows in the network, e.g. the load connected at node four is being supplied locally by the wind farm. On the other hand, the PMSG-based wind farm is providing voltage support, resulting in a voltage magnitude of $1 \mathrm{pu}$ at the low-voltage side of the wind farm transformer even when the TCSC is set in operation to increase the power transfer to $20 \mathrm{MW}$ in the line connecting nodes 4 and 5. The wind generators and TCSC state variables estimated by the NR algorithm for each scenario are the following: scenario (b) $V_{g s c}=1.017 \mathrm{pu}, \delta_{g s c}=9.071^{\circ}$, and scenario (c) $V_{g s c}=1.016 \mathrm{pu}, \delta_{g s c}=8.862^{\circ}, \alpha_{T C S C}=143.499^{\circ}$.

\section{Discussion}

This chapter has put forward the NR-based power flow algorithm which is capable of computing the steady-state operating point of electric networks containing WECS and FACTS devices. The solution problem is formulated in a single-frame of reference, resulting in an efficient iterative solution. Additionally, a PMSG-based wind generator model for power flow studies is presented, which allows for direct voltage magnitude control at the highvoltage side of the wind generator transformer. Numerical examples have shown that FACTS controllers are a practical alternative to integrate WECS into power systems without degrading their operative performance.

\section{Appendix}

- TCSC parameters:

$C_{1}=\frac{X_{C}+X_{L C}}{\pi}, C_{2}=\frac{4 X_{L C}^{2}}{\pi X_{L}}, X_{L C}=\frac{X_{C} X_{L}}{X_{C}-X_{L}}, \omega=\left(\frac{X_{C}}{X_{L}}\right)^{1 / 2}$.

- FSWG parameters:

$A=R_{2}^{2}\left(X_{1}+X_{m}\right), B=\left(X_{2}+X_{m}\right)\left[X_{2} X_{m}+X_{1}\left(X_{2}+X_{m}\right)\right], C=R_{1} R_{2}, D=X_{2} X_{m}+X_{1}\left(X_{2}+X_{m}\right)$,

$E=R_{2}\left(X_{1}+X_{m}\right), \quad F=R_{1}\left(X_{2}+X_{m}\right), H=R_{2} X_{m}^{2}, K=R_{1} R_{2}^{2}, L=R_{1}\left(X_{2}+X_{m}\right)^{2}$,

$M=X_{m} R_{2}\left(X_{1}+X_{m}\right), N=X_{m} R_{1}\left(X_{2}+X_{m}\right), T=R_{1} R_{2} X_{m}, W=X_{m}\left[X_{2} X_{m}+X_{1}\left(X_{2}+X_{m}\right)\right]$.

- SSWG parameters:

$A^{\prime}=\left(X_{1}+X_{m}\right), C^{\prime}=R_{1}, \quad E^{\prime}=\left(X_{1}+X_{m}\right), H^{\prime}=X_{m^{\prime}}^{2} K^{\prime}=R_{1}, M^{\prime}=X_{m}\left(X_{1}+X_{m}\right), T^{\prime}=R_{1} X_{m}$.

The data for each wind farm is (on a base power of 100 MVA): wind farm step-up transformer impedance is $0.2 \mathrm{pu}$, and the impedance of each wind generator transformer is $4.1667 \mathrm{pu}$. Also, the data of each wind generator model are given in Table 4 


\begin{tabular}{llll}
\hline & SR-FSWG & PR-FSWG & SSWG \\
\hline$Z_{1}$ & $0.0027+j 0.025$ & $0.0+j 0.09985$ & $0.00269+j 0.072605$ \\
$Z_{2}$ & $0.0022+j 0.046$ & $0.00373+j 0.10906$ & $0.002199+j 0.04599$ \\
$Z_{m}$ & $j 1.38$ & $j 3.54708$ & $j 1.37997$ \\
Vnom & 690 & 690 & 690 \\
Pnom & 900 & 600 & 1000 \\
\hline
\end{tabular}

Table 4. Wind generator data.

where $Z_{1}$ is the stator impedance $[\Omega], Z_{2}$ is the rotor impedance $[\Omega], Z_{m}$ is the magnetizing impedance [ $\Omega], V_{\text {nom }}$ is the rated voltage $[\mathrm{V}]$ and $P_{\text {nom }}$ is the rated power of the wind generator $[\mathrm{kW}]$.

The coefficients of Equation (10) are as follows: $c_{1}=0.5 ; c_{2}=116 ; c_{3}=0.4 ; c_{4}=0.0 ; c_{5}=0 ; c_{6}=$ $5 ; c_{7}=21 ; c_{8}=0.08$; and $c_{9}=0.035 ; \beta=0$. Also, for the PMSG-based wind generator, its rated voltage is $690 \mathrm{~V}$, and its rated power is $2000 \mathrm{~kW}$.

\section{Acknowledgements}

The authors gratefully acknowledge the financial support granted to MSc. Luis M. Castro by the Consejo Nacional de Ciencia y Tecnología (CONACYT) México, and the University of Michoacán (U.M.S.N.H) for allowing him to undertake PhD studies. The authors gratefully acknowledge the financial support granted to Fuerte-Esquivel CR and Angeles-Camacho C by the FI and the II at the UNAM under the research project 2102.

\section{Author details}

E. Barrios-Martinez ${ }^{1}$, L.M. Castro' ${ }^{2}$, C.R. Fuerte-Esquivel ${ }^{1,2}$ and C. Angeles-Camacho ${ }^{1}$ 1 Instituto de Ingeniería, Universidad Nacional Autónoma de México, UNAM, México

2 Universidad Michoacana de San Nicolás de Hidalgo, UMSNH, Michoacán, México

\section{References}

[1] Acha E, Fuerte-Esquivel CR, Ambriz-Perez H, Angeles-Camacho C. FACTS : Modelling and Simulation in Power Networks, Chichester: John Wiley \& Sons; 2004.

[2] Hwang PI, Ahn SJ, Moon SI. Modeling of the Fixed Speed Wind Turbine Generator System for DTS. IEEE PES General Meeting, 2008: 1-7. 
[3] Burnham DJ, Santoso S, Muljadi E. Variable Rotor-Resistance Control of Wind Turbine Generators. IEEE PES General Meeting, 2009: 1-6.

[4] Senjyu T, Yona A, Funabashi T. Operation Strategies for Stability of Gearless Wind Power Generation Systems. IEEE PES General Meeting, 2008: 1-7.

[5] Castro LM, Fuerte-Esquivel CR, Tovar-Hernández JH. A Unified Approach for the Solution of Power Flows in Electric Power Systems Including Wind Farms. Electric Power Systems Research, in press.

[6] Ackerman T. Wind Power in Power Systems, $1^{\text {st }}$ ed. Chichester: John Wiley \& Sons; 2005.

[7] Bianchi FD, De Battista H, Mantz RJ. Wind Turbine Control Systems - Principles, Modelling and Gain Scheduling Design, $1^{\text {st }}$ ed. London: Springer-Verlan; 2006.

[8] Divya KC, Rao PSN. Models for wind turbine generating systems and their application in load flow studies. Electric Power Systems Research 2006: 76:844-856.

[9] Hong-Woo K, Sung-Soo K, Hee-Sang K. Modeling and control of PMSG-based variable-speed wind turbine. Electric Power Systems Research 2010:80:46-52. 